Edition KWV 
Die „Edition KWV“ beinhaltet hochwertige Werke aus dem Bereich der Wirtschaftswissenschaften. Alle Werke in der Reihe erschienen ursprünglich im Kölner Wissenschaftsverlag, dessen Programm Springer Gabler 2018 übernommen hat.

Weitere Bände in der Reihe http://www.springer.com/series/16033 
Wolfgang H. Schulz

\section{Industrieökonomik und Transportsektor}

Marktdynamik und Marktanpassungen im Güterverkehr

Springer Gabler 
Wolfgang H. Schulz

Wiesbaden, Deutschland

Bis 2018 erschien der Titel im Kölner Wissenschaftsverlag, Köln

Habilitationsschrift Universität zu Köln, 2004

\section{Edition KWV}

ISBN 978-3-658-24687-7 ISBN 978-3-658-24688-4 (eBook)

https://doi.org/10.1007/978-3-658-24688-4

Die Deutsche Nationalbibliothek verzeichnet diese Publikation in der Deutschen Nationalbibliografie; detaillierte bibliografische Daten sind im Internet über http://dnb.d-nb.de abrufbar.

\section{Springer Gabler}

(C) Springer Fachmedien Wiesbaden GmbH, ein Teil von Springer Nature 2004, Nachdruck 2019

Ursprünglich erschienen bei Kölner Wissenschaftsverlag, Köln, 2004

Das Werk einschließlich aller seiner Teile ist urheberrechtlich geschützt. Jede Verwertung, die nicht ausdrücklich vom Urheberrechtsgesetz zugelassen ist, bedarf der vorherigen Zustimmung des Verlags. Das gilt insbesondere für Vervielfältigungen, Bearbeitungen, Übersetzungen, Mikroverfilmungen und die Einspeicherung und Verarbeitung in elektronischen Systemen.

Die Wiedergabe von allgemein beschreibenden Bezeichnungen, Marken, Unternehmensnamen etc. in diesem Werk bedeutet nicht, dass diese frei durch jedermann benutzt werden dürfen. Die Berechtigung zur Benutzung unterliegt, auch ohne gesonderten Hinweis hierzu, den Regeln des Markenrechts. Die Rechte des jeweiligen Zeicheninhabers sind zu beachten.

Der Verlag, die Autoren und die Herausgeber gehen davon aus, dass die Angaben und Informationen in diesem Werk zum Zeitpunkt der Veröffentlichung vollständig und korrekt sind. Weder der Verlag, noch die Autoren oder die Herausgeber übernehmen, ausdrücklich oder implizit, Gewähr für den Inhalt des Werkes, etwaige Fehler oder Äußerungen. Der Verlag bleibt im Hinblick auf geografische Zuordnungen und Gebietsbezeichnungen in veröffentlichten Karten und Institutionsadressen neutral.

Springer Gabler ist ein Imprint der eingetragenen Gesellschaft Springer Fachmedien Wiesbaden GmbH und ist ein Teil von Springer Nature

Die Anschrift der Gesellschaft ist: Abraham-Lincoln-Str. 46, 65189 Wiesbaden, Germany 


\section{Pro Praefatione}

Meliores, prudentiores, constantiores nos tempus in dies reddit. - Libros meos agedum inspiciamus! Libro in primo et altero de fundamentis disseritur. Etiamsi libro in tertio rem diligentius exponam - quod intellego tamen tantum necessaria explico. In proximo libro calculationes comprensae sunt. Id maximo in libro quinto mihi propositum est, ut de agitationes disseram. Ita iam Cicero constituit: "Solum igitur quod sese movet, quia numquam deseritur a se, numquam ne moveri quidem desinit, quin etiam ceteris quae moventur hic fons hoc principium est movendi." Denique rationes in libro sexto comprehensi sunt. Et hostium armatorum totiens non territus, qui possum vestro moveri, ut Velleius Paterculus Scipionem conloquentem facit. 


\section{Inhaltsverzeichnis}

$\begin{array}{ll}\text { Tabellenverzeichnis } & \mathbf{X}\end{array}$

Abbildungsverzeichnis XIV

$\begin{array}{lll}\text { Symbolverzeichnis } & \text { XIX }\end{array}$

$1 \quad$ Problemstellung und Zielsetzungen 1

2 Industrieökonomische Methodik 8

2.1 Ansatzpunkte der industrieökonomischen Analyse 8

$\begin{array}{lll}2.2 & \text { System-dynamische Analyse } & 17\end{array}$

$3 \quad$ Zur Begründung des industrieökonomischen Ansatzes 24

3.1 Bedeutung des Güterverkehrs 26

3.1.1 Wachstum, Verkehrsleistungen und Fahrleistungen 27

3.1.2 Produktivitäts- und Beschäftigungseffekte 33

3.1.3 Regionaler und sektoraler Strukturwandel 35

3.2 Evolutorischer Markt 39

3.2.1 Deregulierung, Bestreitbarkeit und Preiseffekte 40

3.2.2 Intermodaler Wettbewerb 45

$\begin{array}{lll}\text { 3.2.3 Wirtschaftliche Integration } & 47\end{array}$

3.3 Rolle des Staates 48

3.3.1 Infrastrukturpolitik 51

3.3.2 Marktaktivitäten des Staates 55

3.4 Markttheoretische Einordnung des Straßengüterverkehrs 58

3.4.1 Vollständige oder unvollständige Konkurrenz? 59

3.4.2 Die Relevanz externer Nutzen 65

3.4.3 Pekuniäre externe Nutzen des Straßengüterverkehrs 73 
3.4.4 Technologische Externalitäten des Straßengüterverkehrs 83

3.4.5 Verkehrspolitische Relevanz der externen Nutzen 89

$\begin{array}{lll}3.5 & \text { Zusammenfassung } & 91\end{array}$

$4 \quad$ Datengrundlage der industrieökonomischen Analyse $\quad 94$

$\begin{array}{lll}4.1 & \text { Datenlage } & 94\end{array}$

4.2 Probleme bei der Datenerhebung 95

4.3 Wettbewerbssituation auf dem Güterverkehrsmarkt 100

4.4 Intramodaler Wettbewerb 106

4.4.1 Binnenländische Anbieterstruktur 106

4.4.2 Markteintritte und Marktaustritte 112

4.4.3 Europäische Transportunternehmen 116

4.4.4 Transportunternehmen aus den MOE-Staaten 125

4.5 Unternehmensklassen im Straßengüterverkehr 126

$\begin{array}{lll}\text { 4.5.1 Beschäftigungsstruktur } & 127\end{array}$

4.5.2 Erlösstrukturen 131

4.5.3 Kostenstrukturen 133

$\begin{array}{lll}\text { 4.5.4 Gewinnstruktur } & 144\end{array}$

4.6 Struktur des Verkehrsleistungsangebots 145

4.6.1 Das Problem der optimalen Kapazitäten im Straßengüter- 145 verkehr

$\begin{array}{lll}\text { 4.6.2 } & \text { Fahrzeugbestände } & 149\end{array}$

4.6.3 Verkehrsleistungen, Ladekapazitäten und Auslastungsent- 160 wicklung

$\begin{array}{lll}4.7 & \text { Preisstruktur und Preisbildung } & 164\end{array}$

$\begin{array}{lll}4.8 & \text { Ergebnisse der Marktstrukturanalyse } & 167\end{array}$ 
5 System-dynamische Analyse des Straßengüterverkehrsmarktes

5.1 Bildung der Szenarien

5.2 Simulationszeitraum

5.3 Modellierung des Marktverhaltens 185

5.3.1 Kapazitätsdeterminanten, maximales und tatsächliches 185 Kapazitätsangebot

5.3.2 Zuteilung der Verkehrsleistungsnachfrage 193

5.3.3 Gewinn- und Kostenfunktionen 194

5.3.4 Preissetzungsverhalten der Transportunternehmen 196

5.3.5 Veränderungen der Angebotskapazitäten 199

5.3.6 Modellierung der Markteintritte und Marktaustritte 202

5.3.7 Unternehmensinterne Anpassungen der gewichtsmäßigen 206 Fahrzeugauslastung

5.3.8 Ausbauzustand der Straßenverkehrsinfrastruktur 211

5.4 Ergebnisse der Simulationsrechnungen 214

5.4.1 Übersicht über den Simulationsalgorithmus 214

5.4.2 Szenario I: Angebotsüberhang - status quo 221

5.4.3 Szenario II: Einführung Lkw-Maut 237

5.4.4 Szenario III: Kapazitäts-, Auslastungs- und Preisanpassungen 245

5.4.5 Szenario IV: Intermodale Verlagerungen 253

5.4.6 Szenario V: Unterinvestitionen in die Straßeninfrastruktur 263

$\begin{array}{lll}5.5 & \text { Marktentwicklungsaussagen } & 271\end{array}$

$6 \quad$ Schlußfolgerungen $\quad 292$

$\begin{array}{ll}\text { Literaturverzeichnis } & 301\end{array}$ 


\section{Tabellenverzeichnis}

Tabelle 1: $\quad$ Bruttowertschöpfung und Erwerbstätige des Verkehrs- $\quad 34$ sektors in Deutschland

Tabelle 2: $\quad$ Güterverkehrsmarkt in Deutschland (1998) 101

Tabelle 3: Veränderungen der durchschnittlichen Modal Split-An- 104 teile in v.H.

Tabelle 4: $\quad$ Veränderungen der Modal Split-Anteile von 1990 bis 105 1999 in v.H.

Tabelle 5: Anzahl der Unternehmen des Straßengüterverkehrs im 108 Jahr 1998

Tabelle 6: Unternehmen des gewerblichen Straßengüterverkehrs 108 nach unternehmerischem Schwerpunkt von 1994 bis 1998

Tabelle 7: $\quad$ Insolvenzen von Transportunternehmen von 1991 bis 113 1998

Tabelle 8: Güterverkehrsleistungen in der EU-15 von 1991 bis 1998 (in Mrd. tkm)

Tabelle 9: Kabotageverkehrsleistungen nach Quell- und Zielbeziehungen für 1995

Tabelle 10: Kabotageintensitäten zwischen deutschen und ausländischen Transportunternehmen (Juli 1994 bis Juli 1995)

Tabelle 11: Verkehrsleistungen und Verkehrsleistungsanteile ausländischer Transportunternehmen am deutschen Straßengüterverkehr von 1997 bis 1999

Tabelle 12: Transportunternehmen nach Anzahl der Beschäftigten im Jahr 1998

Tabelle 13: Gewerbliche Transportunternehmen des Nah- und 128 Fernverkehrs nach Anzahl der Beschäftigten im Jahr 1998 
Tabelle 14: Anzahl und Struktur der Beschäftigten im Straßengüterverkehr im Jahr 1998

Tabelle 15: Durchschnittliche Umsätze der Unternehmen des gewerblichen Straßengüterverkehrs nach Anzahl der Beschäftigten im Jahr 1998

Tabelle 16: Entwicklung der Gesamtumsätze im gewerblichem Straßengüterverkehr nach Nah- und Fernverkehr von 1995 bis 1998 in Tausend Euro

Tabelle 17: Umsätze im Straßengüterfernverkehr nach Beschäftigten im Jahr 1998

Tabelle 18: Ländervergleich der Lkw-Abgabenbelastung im Jahr 2000 (in Euro pro Jahr)

Tabelle 19: Lkw-Gebührensätze für die Benutzung von Bundesautobahnen in Deutschland (in Euro)

Tabelle 20: Jährliche Mehrbelastung eines Lkw durch die Dieselpreiserhöhung (August 2000 im Vergleich zum Januar 2000)

Tabelle 21: Anteile der Kostenarten am jährlichen Umsatz im ge140 werblichen Straßengüterfernverkehr nach Anzahl der Beschäftigten im Jahr 1998 in v.H.

Tabelle 22: Durchschnittliche Kosten je Unternehmen im Straßen141 güterfernverkehr nach Unternehmensklassen für das Jahr 1998 (in Euro)

Tabelle 23: Plausibilitätsüberprüfung der Position „Kraftstoff- und 143 Schmierstoffkosten“(1998)

Tabelle 24: Größe des Fuhrparks nach Unternehmensklassen im Jahr 1998

Tabelle 25: Durchschnittliche Erlöse, Kosten und Gewinne nach Unternehmensklassen im Jahr 1998 (in Euro)

Tabelle 26: Unternehmen des Straßengüterverkehrs nach Größe des Fuhrparks im Jahr 1998 
Tabelle 27: Veränderungen der Anzahl von Transportunternehmen nach Größe des Fuhrparks von 1998 gegenüber 1994

Tabelle 28: Bestand und Bestandsstruktur der im Straßengüterverkehr eingesetzten Lkw im Jahr 1998

Tabelle 29: Anteile des Fahrzeugbestandes im gewerblichen

Straßengüterverkehr nach Nah- und Fernverkehr in v.H. (1998)

Tabelle 30: Fahrzeugbestände im Straßengüterfernverkehr nach Marktsegmenten im Jahr 1998

Tabelle 31: Anteile der Fahrzeugarten am Fahrzeugbestand im Straßengüterfernverkehr im Jahr 1998 in v.H.

Tabelle 32: Fahrzeugbezogene Investitionen (1998)

Tabelle 33: Verkehrsleistungen nach Unternehmensklassen (1998) 161

Tabelle 34: Ladekapazitäten im Straßengüterverkehr (1998) 162

Tabelle 35: Transportpreise nach Unternehmensklassen in $1998 \quad 164$

Tabelle 36: Preisentwicklung im Straßengüterverkehr seit der Dere- 165 gulierung von 1994 bis 1998

Tabelle 37: Prozentuale Abweichung der Transportpreise von den 166 Kosten je Verkehrsleistung (1998)

Tabelle 38: $\quad$ Strukturdaten der Unternehmensklassen für das Jahr 1998168

Tabelle 39: Zuordnung von system-endogenen und system-exoge- 177 nen Marktänderungen zu den Szenarien

Tabelle 40: $\quad$ Kapazitätsdeterminanten im Jahr 1998

Tabelle 41: Kostensätze nach Unternehmensklassen für 1998 (in 196 Euro)

Tabelle 42: $\quad$ Strukturkoeffizienten der Unternehmensklassen für das 203 Jahr 1998 
Tabelle 43: Zuordnung der Simulationsmodule zu den Szenarien

Tabelle 44: Marktsituation im Jahr 2023

Tabelle 45: Veränderungen der Strukturdaten 2023 gegenüber der Ausgangssituation 1998 für das Szenario I in v.H.

Tabelle 46: Entwicklung der Transportpreise über den Simulations- 260 zeitraum (Szenario IV)

Tabelle 47: $\quad$ Vergleich der Transportpreise im Szenario V mit Sze- 269 nario IV für das Jahr 2023

Tabelle 48: Verkehrsleistungen und Fahrleistungen des Straßen280 güterfernverkehrs im Jahr 2015 - Ergebnisse der Prognosen und Szenarien I, III, IV und V

Tabelle 49: Angebotsüberhang in den Jahren 1998, 2015 und 2023

Tabelle 50: Marktanteile der Unternehmensklassen am Ende des 284 Simulationszeitraums (2023) in v.H.

Tabelle 51: Anzahl der Transportunternehmen nach Unternehmens286 klassen im Jahr 1998 und am Ende des Simulationszeitraums im Jahr 2023

Tabelle 52: Veränderung der Gewinne je Unternehmen nach Unter289 nehmensklassen im Jahr 2023 gegenüber der Ausgangssituation im Jahr 1998 in v.H.

Tabelle 53: Veränderungen der Gesamtgewinne im Straßengüter290 fernverkehr nach Unternehmensklassen im Jahr 2023 gegenüber der Ausgangssituation im Jahr 1998 in v.H. 


\section{Abbildungsverzeichnis}

Abbildung 1: Interdependenzen von Verkehrsleistungsangebot und Verkehrsleistungsnachfrage im Straßengüterverkehr

Abbildung 2: Analytische Vorgehensweise zur Erfassung der Marktdynamik und der Marktanpassungen

Abbildung 3: Dynamisches System

Abbildung 4: Bruttoinlandsprodukt (in Preisen von 1995) und Güterverkehrsleistungen in Deutschland (bis 1990 nur alte Bundesländer, ab 1990 alte und neue Bundesländer) von 1960 bis 1999 (Indexwerte, $1960=100$ )

Abbildung 5: Güterverkehrsleistungen in Deutschland von 1950 bis 1999 in Mrd. tkm nach Verkehrsträgern

Abbildung 6: Transportintensität aller Wirtschaftsbereiche in

Deutschland nach Verkehrsträgern (Indexwerte, 1991=100)

Abbildung 7: Entwicklung der Verkehrsleistung und Fahrleistung im Straßengüterverkehr in Deutschland 1970 bis 1998 (Indexwerte, $1970=100)$

Abbildung 8: $\quad$ Staatliche Ansatzpunkte im Güterverkehr

Abbildung 9: $\quad$ Modernitätsgrad der Schienen-, Straßen- und

Wasserstraßeninfrastruktur (1965-1999)

Abbildung 10: Technologische Externalitäten zwischen zwei Industrien

Abbildung 11: Durchschnittliche Entwicklung der Modal Split-An103 teile im Güterverkehr von 1950 bis 1999 in Prozent

Abbildung 12: Modal Split-Entwicklung im Güterverkehr von 1990 bis 1999 in v.H. 
Abbildung 13: Entwicklung der durchschnittlichen Fern- und

Nahverkehrsanteile im Straßengüterverkehr von

1950 bis 1999 in Prozent

Abbildung 14: Durchschnittliche Verkehrsleistungsanteile im

Straßengüterfernverkehr von 1950 bis 1999 in

Prozent

Abbildung 15: Verkehrsleistungsentwicklung im Straßengüter- 111 fernverkehr von 1990 bis 1998 in Mrd. tkm

Abbildung 16: Vergleich der Unternehmensinsolvenzen im Wirt- 114 schaftszweig Verkehr mit den anderen Wirtschaftszweigen (Indexwerte, 1991=100)

Abbildung 17: Position des deutschen Straßengüterverkehrs in der EU im Jahr 1998 (Anteile an den Verkehrsleistungen in v.H.)

Abbildung 18: Struktur der Beschäftigten im gewerblichen

Straßengüterverkehr nach Größe des Fuhrparks (1998)

Abbildung 19: $\quad$ Struktur der Beschäftigten im Werkverkehr nach Größe des Fuhrparks (1998)

Abbildung 20: Vorgehensweise zur Ermittlung der Fahrzeugbestände im gewerblichen Straßengüterfernverkehr für das Jahr 1998

Abbildung 21: Entwicklung der Fahrzeugauslastung im Straßengüterverkehr von 1980 bis 1999 in v.H.

Abbildung 22: Zusammenhang zwischen gewichtsmäßiger Auslastung und Verkehrsleistung im gewerblichen Straßengüterverkehr (1980 bis 1998)

Abbildung 23: Vergleich zwischen linearer Fortschreibung und 210 hyperbolischer Entwicklung der gewichtsmäßigen Fahrzeugauslastung im Straßengüterfernverkehr für den Zeitraum von 1998 bis 2023

Abbildung 24: Simulationsmodule 
Abbildung 25: Übersicht über den Simulationsablauf für Szenario I 222

Abbildung 26: Entwicklung der Fahrleistungen, des Verkehrs- 225 leistungsangebots und der Verkehrsleistungsnachfrage (Szenario I, Indexwerte, 1998=100)

Abbildung 27: Entwicklung der Marktaustritte von 1998 bis $2023 \quad 229$ (Szenario I, Anzahl der Unternehmen, absolut)

Abbildung 28: Anzahl der Transportunternehmen nach Unterneh- 230 mensklassen in den Jahren 1998 und 2023 (absolut)

Abbildung 29: Fuhrparkgrößen der Unternehmensklassen D, E 232 und F in den Jahren 1998 und 2023 (Szenario I, absolut)

Abbildung 30: Entwicklung des Verkehrsleistungsangebots nach 234 Unternehmensklassen von 1998 bis 2023 (Szenario I, in Mrd. tkm)

Abbildung 31: Veränderung der gesamten Kosten (Szenario I, 235 in v.H.)

Abbildung 32: Veränderung der Gewinne nach Unternehmens- 236 klassen (Szenario I, in v.H.)

Abbildung 33: Übersicht über den Simulationsablauf für Szenario II 238

Abbildung 34: Veränderung der Kosten infolge der fahrleistungs- 239 abhängigen Straßenbenutzungsgebühr (Szenario II, in v.H.)

Abbildung 35: Markteintritte und Marktaustritte infolge der fahr- 240 leistungsabhängigen Straßenbenutzungsgebühr von 1999 bis 2023 (Szenario II, Anzahl der Unternehmen, absolut)

Abbildung 36: Gewinnvergleich zwischen Szenario I (ohne Lkw- 241 Maut) und Szenario II (mit Lkw-Maut) für das Jahr 2003

Abbildung 37: Kauf zusätzlicher Lkw ohne und mit Lkw-Maut 242 im Jahr 2004 (absolut) 
Abbildung 38: Entwicklung der Gewinne und Kosten nach Ein- 244 führung der Lkw-Maut im gesamten Straßengüterfernverkehr in Mio. Euro (Szenario II)

Abbildung 39: Übersicht über den Simulationsablauf für Szenario III 246

Abbildung 40: $\quad$ Entwicklung der Fahrleistungen, der Verkehrs- 247 leistungen und der Verkehrsleistungskapazitäten (Szenario III, Indexwerte, $1998=100$ )

Abbildung 41: $\quad$ Marktaustritte (Szenario III, Anzahl der Unterneh- 248 men, absolut)

Abbildung 42: Gewinnentwicklung in den Unternehmensklassen 249 A, C, D, E und F in v.H. (Szenario III, in v.H.)

Abbildung 43: Gewinn- und Verlustentwicklung für die Unter- 251 nehmensklasse B in Euro (Szenario III)

Abbildung 44: $\quad$ Aufteilung der Verkehrsleistungsnachfrage auf die 252 Unternehmensklassen (Szenario III, in Mrd. tkm)

Abbildung 45: Veränderung der Transportpreise nach Unterneh- 253 mensklassen in v.H. (Szenario III)

Abbildung 46: Überblick über den Simulationsablauf für Szenario IV 255

Abbildung 47: Entwicklung der Fahrleistungen, der Verkehrs- 258 leistungen und der Verkehrsleistungskapazitäten ohne Marktaustritte und Markteintritte (Szenario IV, Indexwerte, $1998=100$ )

Abbildung 48: Entwicklung der Fahrleistungen, der Verkehrsleistungen und der Verkehrsleistungskapazitäten mit Markteintritten und Marktaustritten (Szenario IV, Indexwerte, 1998=100)

Abbildung 49: $\quad$ Entwicklung der Markteintritte und Marktaustritte 259 von 1999 bis 2023 (Szenario IV, Anzahl der Unternehmen, absolut)

Abbildung 50: Preisveränderungen nach Unternehmensklassen in 261 v.H. (Szenario IV) 
Abbildung 51: Entwicklung des Verkehrsleistungsangebots nach 262 Unternehmensklassen (Szenario IV, in Mrd. tkm)

Abbildung 52: Veränderung der Gesamtkosten nach Unterneh- 262 mensklassen (Szenario IV, in v.H.)

Abbildung 53: Überblick über den Simulationsablauf für Szenario V 265

Abbildung 54: Entwicklung der Fahrleistungen, der Verkehrs- 267 leistungen und der Verkehrsleistungskapazitäten (Szenario V, Indexwerte, $1998=100$ )

Abbildung 55: Vergleich der Fahrleistungsentwicklung von 1998267 bis 2023 für die Szenarien IV und V in Mrd. Fz-km

Abbildung 56: Vergleich der gesamten Markteintritte und Markt- 268 austritte zwischen Szenario IV und Szenario V (Anzahl der Unternehmen, absolut)

Abbildung 57: Entwicklung der gewichtsmäßigen Fahrzeugaus- 282 lastung in den Szenarien von 1998 bis 2023 (in v.H.)

Abbildung 58: Relative Konzentration im Straßengüterfernverkehr 287 


\section{Symbolverzeichnis}

$\mathrm{AK}_{\mathrm{t}} \quad$ marktrelevantes Gesamtangebot an Verkehrsleistungskapazität aller Unternehmensklassen für den gesamten Straßengüterfernverkehr in der Zeitperiode t.

$\mathrm{AK}_{\mathrm{t}}^{\max } \quad$ maximales Gesamtangebot an Verkehrsleistungskapazität aller Unternehmensklassen in der Zeitperiode t.

$\mathrm{B}_{\mathrm{i}, \mathrm{t}} \quad$ Anzahl der Beschäftigten eines Transportunternehmens i einer Unternehmensklasse $U$ in der Zeitperiode $t$.

BS

Binnenschiffahrt.

$\mathrm{C}_{\mathrm{i}, \mathrm{t}} \quad$ gesamte Kosten eines Transportunternehmens i einer Unternehmensklasse $\mathrm{U}$ in der Zeitperiode t.

$c_{1, i, t} \quad$ Personalkostensatz eines Transportunternehmens i einer Unternehmensklasse $\mathrm{U}$ in der Zeitperiode $\mathrm{t}$.

$\mathrm{c}_{2, \mathrm{i}, \mathrm{t}} \quad$ Kraft- und Schmierstoffkostensatz eines Transportunternehmens i einer Unternehmensklasse $U$ in der Zeitperiode t.

$\mathrm{c}_{3, \mathrm{i}, \mathrm{t}} \quad$ Wartungskostensatz eines Transportunternehmens i einer Unternehmensklasse $\mathrm{U}$ in der Zeitperiode t.

$\mathrm{c}_{4, \mathrm{i}, \mathrm{t}} \quad$ Versicherungskostensatz eines Transportunternehmens i einer Unternehmensklasse $\mathrm{U}$ in der Zeitperiode t.

$c_{5, i, t} \quad$ Kfz-Steuersatz eines Transportunternehmens i einer Unternehmensklasse $\mathrm{U}$ in der Zeitperiode $\mathrm{t}$.

$\mathrm{c}_{6, \mathrm{i}, \mathrm{t}} \quad$ Fremdleistungskostensatz eines Transportunternehmens i einer Unternehmensklasse $\mathrm{U}$ in der Zeitperiode t.

$\mathrm{C}_{\mathrm{KE}, \mathrm{t}} \quad$ Anschaffungskosten für eine Kapazitätseinheit in der Zeitperiode $t$.

$D_{t} \quad$ Verkehrsleistungsnachfrage in der Zeitperiode $t$.

DLkw $_{u, t} \quad$ durchschnittliche Anzahl der Fahrzeuge je Transportunternehmen $\mathrm{i}$ in einer Unternehmensklasse $\mathrm{U}$ in der Zeitperiode $\mathrm{t}$. 
$\mathrm{d}_{\mathrm{u}, \mathrm{t}} \quad$ Marktnachfrage, die in der Zeitperiode $\mathrm{t}$ auf die Transportunternehmen i einer Unternehmensklasse U entfällt.

$\mathrm{d}_{\mathrm{i}, \mathrm{t}} \quad$ erbrachte Verkehrsleistung in tkm eines Transportunternehmens i einer Unternehmensklasse $U$ in der Zeitperiode $t$.

E Eisenbahn.

F Anzahl der Transportfälle pro Jahr (Fahrten pro Kapazitätseinheit).

$F^{\max } \quad$ maximale Anzahl der Transportfälle einer Kapazitätseinheit pro Jahr.

$\mathrm{Fl}_{\mathrm{i}, \mathrm{t}} \quad$ erbrachte Fahrleistungen (Fahrzeugkilometer) eines Transportunternehmens i einer Unternehmensklasse $U$ in der Zeitperiode $\mathrm{t}$.

FL Fahrleistungen in Fahrzeugkilometern [Fz-km].

FL $\quad$ Fahrleistungen einer Kapazitätseinheit in Kilometern pro Jahr.

Fz-km Fahrzeugkilometer.

gon

Neugrad $($ rechter Winkel $=100$ gon, Vollwinkel $=400$ gon $)$.

$\mathrm{K}_{\mathrm{u}, \mathrm{t}}^{\max } \quad$ maximale Verkehrsleistungskapazität aller Transportunternehmen einer Unternehmensklasse $\mathrm{U}$ mit $\mathrm{U}=\mathrm{A}, \ldots, \mathrm{F}$ in der Zeitperiode $t$.

$\mathrm{K}_{\mathrm{U}, \mathrm{t}} \quad$ tatsächlich eingesetzte Verkehrsleistungskapazität aller Transportunternehmen einer Unternehmensklasse $\mathrm{U}$ mit $\mathrm{U}=\mathrm{A}, \ldots, \mathrm{F}$ in der Zeitperiode $\mathrm{t}$.

$\mathrm{K}_{\mathrm{i}, \mathrm{t}} \quad$ erbrachte Kapazitätsleistung eines Transportunternehmens i mit den Kapazitätseinheit j einer Unternehmensklasse U in der Zeitperiode $\mathrm{t}$.

$\mathrm{K}_{\mathrm{i}, \mathrm{m}}^{\max } \quad$ maximale Kapazität in Tonnenkilometern für ein Transportunternehmen i einer Unternehmensklasse U.

KE Kapazitätseinheit. 
$\mathrm{KE}_{\mathrm{i}, \mathrm{j}, \mathrm{j}} \mathrm{j}$-Kapazitätseinheiten eines Transportunternehmens $\mathrm{i}$ einer Unternehmensklasse $\mathrm{U}$ in der Zeitperiode t.

$L_{\mathrm{u}, \mathrm{t}}^{\mathrm{opt}} \quad$ Anzahl der Fahrzeuge in einer Unternehmensklasse $\mathrm{U}$ in der Zeitperiode $t$, die benötigt wird, um die nachgefragte Verkehrsleistung $\mathrm{d}_{\mathrm{u}, \mathrm{t}} \mathrm{zu}$ befördern.

Lt $t_{K E} \quad$ Ladegewicht einer Kapazitätseinheit in Tonnen.

$L_{\mathrm{KE}}^{\max } \quad$ zulässiges Höchstgewicht einer Kapazitätseinheit in Tonnen.

LZ gesetzlich vorgegebene maximale Lenkzeit pro Tag $\left(L^{\max }=\right.$ $10 \mathrm{~h})$.

M Faktor zur Beschreibung des mikroskopischen Verkehrsflußverhaltens $(0 \leq M \leq 1)$, wobei für Lkw mit $v^{z u l}=80 \mathrm{~km} / \mathrm{h}$ ein Wert von $M$ zwischen 0 und 0,25 die Stausituation beschreibt und $M=1$ den störungsfreien Verkehrsfluß abbildet.

ME Marktergebnis (=realisierte Verkehrsleistung in Tonnenkilometern) in der Zeitperiode t.

m Index für die Anzahl der Unternehmen in einer Unternehmensklasse

$\mathrm{p}_{\mathrm{i}, \mathrm{t}} \quad$ Transportpreis eines Transportunternehmens i einer Unternehmensklasse $\mathrm{U}$ in der Zeitperiode $\mathrm{t}$.

$\mathrm{p}_{\mathrm{KE}, \mathrm{t}} \quad$ Wiederverkaufspreis für eine Kapazitätseinheit in der Zeitperiode $\mathrm{t}$.

$\mathrm{Q}_{\mathrm{Lkw}} \quad$ durchschnittliche tägliche Lkw-Verkehrsstärke in Lkw je $24 \mathrm{~h}$.

$Q_{\mathrm{Pkw}} \quad$ durchschnittliche tägliche Pkw-Verkehrsstärke in Pkw je $24 \mathrm{~h}$.

Su

Marktstrukturkoeffizient für eine Unternehmensklasse U.

SG Straßengüterverkehr.

$\mathrm{ST}_{\mathrm{j}} \quad$ Straßentyp (z.B. Bundesautobahn mit 3 Fahrstreifen je Richtung, Bundesfernstraße mit 2 Fahrstreifen je Richtung) mit $\mathrm{j}=$ $1, \ldots, \mathrm{n}$. 
$\mathrm{T}_{u}^{\max } \quad$ maximal mögliche Einsatztage für eine Kapazitätseinheit einer Unternehmensklasse pro Jahr mit $\mathrm{T}_{u}^{\max }=255$ Tage.

$\mathrm{TKM}_{\mathrm{KE}}^{\max }$ maximale Tonnenkilometer einer Kapazitätseinheit in der Zeitperiode $\mathrm{t}$.

$\mathrm{Tkm}_{\mathrm{KE}, \mathrm{t}} \quad$ Verkehrsleistungen einer Kapazitätseinheit in der Zeitperiode $t$.

tkm Tonnenkilometer.

$\mathrm{U} \quad$ Index für Unternehmensklassen A, B, C, D, E und F.

$\mathrm{U}_{\mathrm{u}, 98} \quad$ Anzahl der Transportunternehmen in einer Unternehmensklasse U im Jahr 1998.

$\hat{U}_{\mathrm{U}, \mathrm{t}} \quad$ Anzahl der Transportunternehmen i einer Unternehmensklasse $U$ in der Zeitperiode $t$, die ausreichen um die Verkehrsleistungsnachfrage in einer Unternehmensklasse zu befriedigen.

$\mathrm{U}_{\mathrm{u}, \mathrm{t}}$

tatsächliche Anzahl von Transportunternehmen in einer Unternehmensklasse $\mathrm{U}$ in der Zeitperiode $\mathrm{t}$.

durchschnittliche Lkw-Geschwindigkeit $(\mathrm{km} / \mathrm{h})$.

realisierte Geschwindigkeit einer Kapazitätseinheit in km/h.

empirisch ermittelte Geschwindigkeit eines Lkw in $\mathrm{km} / \mathrm{h}$.

$\mathrm{v}^{\mathrm{zul}}$

gesetzlich zulässige Höchstgeschwindigkeit für Lkw in km/h.

$\mathrm{V}_{\mathrm{KE}}^{\mathrm{zul}, \mathrm{ST}}$

gesetzlich zulässige Höchstgeschwindigkeit für Lkw in Abhängigkeit vom Straßentyp $\mathrm{j}$ in $\mathrm{km} / \mathrm{h}$.

W durchschnittliche Versandweite in km pro Fahrt.

z mögliche Fahrten pro Stunde (Fahrten/h).

$\varepsilon_{\text {qipj }} \quad$ Kreuzpreiselastizität der Verkehrsleistungsnachfrage für die relative Leistungsveränderung des i-ten Verkehrsträgers mit $\mathrm{i}$ $=\mathrm{E}, \mathrm{B}, \mathrm{SG}$ im Verhältnis zur relativen Preisänderung des $\mathrm{j}$-ten Verkehrsträgers mit $j=E, B, S G$, wobei $i \neq j$. 
$\kappa \quad$ Kurvigkeit definiert als die auf die Streckenlänge eines Straßentyps $\mathrm{ST}_{\mathrm{j}}[\mathrm{km}]$ bezogene Summe der Absolutbeträge der Winkeländerungen [gon] des Straßentyps in gon $/ \mathrm{km}$.

$\Pi_{\mathrm{i}, \mathrm{t}} \quad$ Gewinn eines Transportunternehmens i einer Unternehmensklasse U in der Zeitperiode t.

mittlere Längsneigung (=Steigung) eines Straßentyps in v.H.

$\varpi$ Ausbauzustand der Straßeninfrastruktur in Netzkilometern.

$\omega \cup$ Parameter für die gewichtsmäßige Auslastung $\left(0<\omega_{U}<1\right)$ der Kapazitätseinheiten in einer Unternehmensklasse.

$\xi_{u} \quad$ Parameter für die von den Transportunternehmen (U) einer Unternehmensklasse angestrebte zeitliche Auslastung pro Jahr einer Kapazitätseinheit $\left(0<\xi_{U}<1\right)$ zur Ermittlung der Einsatztage einer Kapazitätseinheit.

$\psi_{u} \quad$ Parameter für die von den Transportunternehmen (U) einer Unternehmensklasse angestrebte zeitliche Auslastung einer Kapazitätseinheit pro Tag $\left(0<\psi_{u}<1\right)$ zur Ermittlung der Einsatzstunden einer Kapazitätseinheit pro Tag. 